\title{
The Varied Nature of Risk and Considerations for the Water Industry: A Review of the Literature
}

\author{
Anna Kosovac ${ }^{1}$, Brian Davidson ${ }^{1}$, Hector Malano $^{2} \&$ Julia Cook $^{3}$ \\ ${ }^{1}$ School of Veterinary and Agricultural Sciences, The University of Melbourne, Melbourne, Australia \\ ${ }^{2}$ School of Infrastructure Engineering, The University of Melbourne, Melbourne, Australia \\ ${ }^{3}$ Youth Research Centre, Graduate School of Education, The University of Melbourne, Melbourne, Australia \\ Correspondence: Anna Kosovac, School of Veterinary and Agricultural Sciences, The University of Melbourne, \\ 3010, Melbourne, Australia. E-mail: anna.kosovac@unimelb.edu.au
}

Received: May 15, 2017

doi:10.5539/enrr.v7n2p80
Accepted: May 30, 2017

Online Published: May 31, 2017

\begin{abstract}
In many circumstances within the water industry, project managers act as the gatekeepers for new infrastructure and water projects by way of determining, or at least providing, an assessment of whether to proceed with the project. Their assessments, which are considerably based on risk, disproportionately assume a technical, rational approach. Psychological, sociological and cultural risk approaches provide a comprehensive guide into how risks are assessed, highlighting areas that are not considered by the traditional technical risk approach. This article provides a review of these approaches, and in so doing contends that existing risk assessments in the water industry ignore sociological and psychological contexts in situations, leading to an assessment that may not be reliable.
\end{abstract}

Keywords: cultural studies, risk management, water, sociology, uncertainty

\section{Introduction}

The interest in and subsequent literature within the field of risk has grown exponentially in the previous 30 years, both in research and in its everyday use (Lupton, 2013, p. 8). The very notion of 'risk' is a contemporary concept, with the literature surrounding risk having rapidly expanded, reflecting the added interest in both public and academic spheres (Note 1). Risk research spans numerous disciplinary areas and is prevalent in the academic fields of psychology, engineering and sociology, leading to differing perspectives and approaches. The use of risk perspectives by stakeholders within the water industry has, however, largely been restricted to technical, rational approaches. The aim of this paper is to highlight other risk approaches not usually considered by risk assessors to promote discussion on whether existing technical approaches should remain unquestioned, or whether there may be scope to create an interdisciplinary tool to assess risk more effectively.

This paper provides a concise and thorough review of risk perspectives, and how they differ between disciplines. The review also captures the criticisms of each approach, with the aim of providing an overview of risk-based discourse in the academic sphere. There are different strains of risk theory, but three main variants are explored:

\section{Technical perspective on risk; \\ Sociological and cultural perspectives on risk; and \\ - Psychological perspectives on risk.}

The above elements of risk research are by no means exhaustive, but comprise the most prominent areas of risk research. The technical approach is the one most utilised in the water industry, with its focus on risk probability and risk consequence. Alternative approaches such as the Cultural or Psychological approaches, allow a much broader and more succinct look into how risk is analysed within a particular cultural viewpoint, or due to existing psychological affiliations. They offer a more comprehensive analysis of the risk assessor's societal influences and previous experiences. The aim of the review is to expand the knowledge of those responsible for assessing risk in large-scale engineering water projects. The paper will examine the business as usual technical-economic approach, to a psychological and social approach. The three individual approaches are reviewed with emphasis on the short-comings of the technical-economic approach and the potential insights of 
the social and psychological approaches. The central themes from all three approaches are compared, with the aim of presenting a case for further consideration of how risk is calculated within the water industry.

\section{Risk and Uncertainty}

Risk, as a term, is a relatively recent concept (Note 2) that has seen a significant increase in its use in recent decades (Lupton, 2013, p. 11). Uncertainty underpins the very idea of risk, with Beck defining the term as 'calculable uncertainty'(Beck, 1999, p. 153) and Taylor-Gooby and Zinn similarly claiming risk is a set of "possibilities, chances or likelihoods of events, often as consequences of some activity or policy" (Taylor-Gooby \& Zinn, 2006, p. 1). A key notion in the concept of risk is the implication that there is a component of human control in avoiding unwanted consequences (Zinn, 2009, p. 4). This control morphed into an increase in the use of 'risk management', the widespread tool of varied methods for controlling risks. As is explained further in following sections on risk theory, 'risk' can be interpreted as being wholly objective, or is seen as socially constructed. Indeed, claims to the epistemological status of risk provide a key point of differentiation between the theories.

Understanding risk and how it is constructed is vital in further progressing the field in practice. In the words of Beck it essentially:

... highlights the new power game of risk and its meta-norms: who is to define the riskiness of a product,

a technology, and on what grounds in an age of manufactured uncertainties? (Beck, 1999, p. 4)

Risk theories can work to bring about a greater understanding of the power implied in developing risk assessments and responses. In particular, that the action arising from the perceived risk may change between assessors.

\subsection{Technical Perspectives on Risk}

The technical approach to risk adopts the objective, or positivist approach - viewing risk assessments as an exercise undertaken by experts. The risk assessor first identifies the risks and benefits associated with a project, making an objectified assessment based on certain factors as devised by experts. Many government organisations and regulatory bodies, such as the Environmental Protection Agency in Victoria, Australia, utilise this form of risk theory through the assessment of two inputs: risk consequence and risk probability (Environment Protection Agency, 2004; Victorian Managed Insurance Authority, 2016).

Starr (1969) was the major proponent of this type of objectified risk analysis. He promoted the idea that risk assessments can be used to form an ideal scenario wherein there is a calculated risk to benefit approach (Starr, 1969). This type of assessment relies on legitimate statistical and/or fiscal data being readily available (Renn, 1992).

Other forms include the Cost-Benefit Risk Analysis, which is a central tenet of economic methodology. This approach embraces consideration of possible costs versus benefits when assessing future actions or approaches, with a focus on financial spending and investment. Accordingly, both technical and economic risk assessments rely on the assumption that all decision makers and actors are rational. They utilise Rational Actor Theory when predicting the consequences and actions of others, producing an arguably positivist and objectified approach to risk management (Von Neumann \& Morgenstern, 2007). Rational Actor Theory is based on actors taking action based on predictable, consistent goal setting while also maximising expected utility. This approach is primarily focused on the choice and actions of individuals, and their behaviours in decision making. The rational actor is diametrically opposed to the irrational actor, who is deemed to be spontaneous, random and impulsive. The rational actor is generally assumed to be acting in self-interest (Von Neumann \& Morgenstern, 2007).

Significant criticism has been lodged against this type of risk analysis. For instance, Self's seminal piece on 'Econocracy' refutes the very nature of cost-based risk analyses, and in particular cost-benefit analyses (Self, 1975). He argues that there is an inherent falsehood to these approaches and that they are not an aid to decision making. For Self, the very nature of cost-benefit analyses, and their obsession with costing elements such as welfare and social benefit, is flawed and biased (Self, 1975). Indeed, in an attempt to generate a more objectified and rational approach to decision making, the method instead creates an assessment that is wholly irrational due to its attempt to quantify elements that cannot necessarily be effectively quantified, thus carrying an inherent risk in its use (Beck, 1992). Ulrich Beck, a prominent sociological theorist of risk, proposed that due to limits of rationality in individuals, it cannot be assumed that all are rational actors in their decision making (Beck, 1992). As purely rational actors are fictional, it remains necessary to leave space for an element of subjectivity in the conception of risk. 
In a stronger iteration of Beck's approach, Slovic argues that there is simply no such concept as a 'real risk' or 'objective risk' (Slovic, 1993, p. 690). He argues that in making the assumption that the probability combined with the consequence of risk is objective and that all assessors are rational in their approach to this analysis, it ignores the very nature of a 'perceived risk'. This assertion hinges primarily on the claim that the way in which a layperson will analyse a risk is likely to differ from the approach taken by an expert in that field due to the tools one has on hand. These tools of analysis can be drawn from factors such as education, culture and lived experience in order to determine one's relationship to a risk. The sense of dread associated with a risk is discussed by Slovic (1993), and he contends that this accounts for a strong emotive response to a risk in decision making (Slovic, 1992, p. 121). The higher that factor of 'dread risk', the larger the perceived risk as analysed by the public. For instance, Slovic argues that phenomenon which he terms 'dread risks', which often loom large in the public consciousness due to previous catastrophes (a paradigm example of which would be nuclear power), are likely to be perceived as posing a high level of risk by lay people, even in cases in which this assessment is not reflective of expert opinion. This may not fall in line with experts' assessments of risks when based on a technical risk analysis. This is due to the fact that experts are likely to base their perceptions of risk on potential mortalities or level of harm per capita (Slovic, 1992). The public frequently rejects models of risk devised by scientists using these methods, as they are unlikely to adhere to the value-based perceptions presented by an individual (Gregory \& Mendelsohn, 1993). This is not to state that a particular viewpoint, whether it be the layperson or the expert, is necessarily better than the other, but rather to highlight that there are elements that both groups may not consider that the other group will place importance upon. These risk analyses also fail to take account of social and political elements of risk, and any other areas that can be difficult to measure quantitatively. Therefore, the argument that technical perspectives on risk are risky themselves is apt when considering the enormous effect that social impacts may have on a project/process.

\subsection{Sociological and Cultural Perspectives on Risk}

Social impacts are the key focus of sociological and cultural theories of risk. Scientific knowledge, and reliance on a positivist viewpoint, cannot settle a debate where differing expert views exist (Pielke, 2007, p. 51). Consequently, we have seen a blurring of lines between expert and layperson knowledge (Zinn, 2009).

Beck prescribes to the sociological reading of risk and is one of the key theorists in the field. He argues that new risks have resulted from the progression through modernity, and that they have reconfigured the way risks are perceived (Beck, 1992). Beck describes these new risks as being relatively imperceptible (for example, the effect of radiation), not defined by geographical boundaries (they can affect a very wide populace, despite state borders) and not discriminatory (they affect all classes of people equally) (Beck, 1992). These 'new' risks can have an impact on large numbers of people, and as they are imperceptible, migration, adaptation and avoidance efforts rely on knowledge of the risk (Beck, 1992). For Beck, this knowledge, as well as the way in which it is communicated, is socially constructed.

Indeed, adopting a constructivist approach, Beck concludes that in a modern society, which is increasingly obsessed with managing risks, great political power lies in the ability to define risk and construct meaning in a way that may prioritise certain aspects over others (Beck, 2006). For instance, the very definition of a risk within an assessment of a project can result in whether a project proceeds. In this way, much power can be afforded to the risk assessor and this applies to the water industry. In cultural theories of risk, the role of the media is often cited, particularly in shaping the values and views of individuals. Media outlets mirror the cultural zeitgeist and can as such, represent the prisms through which these risks are viewed (Anderson, 1997). Slovic, Fischoff and Lichtenstein's studies show a strong positive correlation (0.7) between media reporting in the causes of a death and higher public perception of the risk of this cause of death (Fischhoff, Slovic, Lichtenstein, Read, \& Combs, 1978). Furthermore, these studies also indicate that negative news tends to be perceived more credibly by the public than positive news in the media. Parallels exist between Beck's risk society thesis and how the public have moved toward a preoccupation with risk(Beck, 2006).

Mary Douglas, an anthropologist in the field of cultural risk theory, espouses the view that 'what one sees depends on where one stands'(Rayner, 1992, p. 7), such that the definition of risk, and thus power, is dependent on factors such as gender, race, political views/affiliation, emotional affect and trust. The cultural relativist argument accordingly states that all risk evaluators are inherently biased in their judgements.

Furthermore, cultural relativism refutes the technical risk approach's assumption that added knowledge of a hazard renders one's decision more rational. Cultural relativists understand risk assessments as aesthetic judgements, concluding that existing cultural patterns will sway individuals to adopt certain values over others, ultimately affecting the way that they view risks. 
Sociological and cultural risk approaches afford considerable power to actors who are able to define risk, such as media outlets, public figures and experts. These risk definitions are predominantly based on these actor's own beliefs and rationalities (as further defined by the scope of social and cultural norms) and are used to formulate a risk dialogue that can allow powerful actors to maximise risks for others while minimising their impact upon themselves (Beck, 2006). Douglas considers how risk dialogue has changed significantly in the Western world, with less 'quantifying' of risks, and a supplanting of 'unacceptable danger' by risk discourse (Douglas, 1992). The relationship between risk and morality is explored by this theory, in particular, how contemporary moral viewpoints create a guiding principle for how risks are assessed. This is a result of the level of attention afforded to a certain risk, and subsequently, onto whom the responsibility of the risk falls. Douglas also considers that in Western societies, societies/organisations/groups will look for whom to blame for a negative risk:

Under a banner of risk reduction, a new blaming system has replaced the former combination of moralistic condemning the victim and opportunistic condemning the victim's incompetence (Douglas, 1992, p. 26).

Douglas relates this increased interest in risk and blame to large organisations and the nature of globalisation itself. Both the increase in and growing interconnectedness of companies and organisations have propelled the interest in risk among Western populations, and in doing so, prompted individuals to hold these companies to moral principles as defined by social and cultural norms.

Cultural theories of risk do not consider the individual, but instead social aggregates. This presupposes that a member of a social aggregate will assess risk like their peers within the same group. This aspect of the theory is rendered difficult when it is faced with explaining what happens when an individual is a member of many different social aggregates, and how this affects the decisions that they make. This is significant when confronted with social aggregates that have views that are very different, even contradictory, from one another (Renn, 1992, p. 75). Sociological theories, such as those of Beck, focus on the individual who is shaped by personal experiences and culture, and whose perception of risk does not necessarily reflect the cultural groups to which they belong. In spite of this, both sociological and cultural theories generally adhere to a 'weak' constructivist principle, viewing risk as having a basis in material reality, but contending that it can only be understood through the lens of social and cultural context.

\subsection{Psychological Perspective on Risk}

Not unlike the sociological and cultural theorists, Slovic, a key proponent of the psychological risk approach, recognises that 'danger is real, but risk is socially constructed (Slovic, 1993, p. 659)'. Similar to the sociological risk perspective, the psychological view focuses on the individual. The psychological perspective of risk focuses on how individuals form judgements based on their underlying beliefs and experiences. Risk perception is closely tied to an individual's worldview, thus linking risk choices to their own values and psychology (Fischhoff et al., 1978). These worldviews create a personal prism through which the individual assesses risks.

The psychological perspective predominantly focuses on decision-making biases, in particular the intuitive processing of uncertainty. This theory promotes the importance of personal and contextual variables in assigning risk to certain factors over others. The first element of this, as researched by Slovic, Fischoff and Lichtenstein, is the 'availability heuristic' (Fischhoff et al., 1978), referring to a phenomenon that occurs when people are able to judge an event as more likely when they are faced with it, or if it is easier to recall, despite their exposure to any data that may contradict their perceptions (Fischhoff, 1975). For example, a person who has a family member with a particular form of cancer may perceive the rate of this type of cancer to be higher in the general public than is actually the case. This 'availability heuristic' is a key element in assessing risk in the psychological risk perspective. The elevation of particular risks as a result of an individual's awareness or experience of them can influence the resources the individual allocates to ameliorating that risk, compared to other risks they have no personal affiliation with. The scenario creates a disparity in the homogeneity of behaviours towards a particular risk, which can in turn create division and debate, leaving the definition of the risk itself as being open to interpretation. In the case of a government or society, where the pool of resources that stem from each person's contribution is to be allocated, this differing perspective could create heightened tensions that engage citizens in further public debate. Slovic argues that a significant difference in the risk perceptions of the public and policymakers can result in a 'crisis of confidence,' (Slovic, 1992) leading the public to respond to their own risk perceptions rather than risk assessments undertaken by experts or a regulatory body.

The research within the psychological perspective on risk also highlights the perception of personal control in affecting the assessment of risk. People are generally more likely to take risks if it is by their own choice and less likely to accept even a lower level of risk if they feel that it is forced on them. In addition to this, Alhakami and 
Slovic have confirmed an inverse relationship between perceived benefits from a risk versus the perceived risk, and have found it to be a strongly negative correlation (Alhakami \& Slovic, 1994; Siegrist, Cvetkovich, \& Roth, 2000).

If the perceived risk rises, its perceived benefits drop. Tversky and Kahneman (Kahneman \& Tversky, 1979) found similar results, demonstrating that individuals are risk averse if the potential losses are high and risk prone if the stakes for gains are high. Therefore, risk aversion is seen as closely correlated to personal preferences and beliefs (Fischhoff et al., 1978, pp. 140-142).

This theory also takes into account the role of emotions in decision making, an area that has only been seriously considered by sociologists analysing risk relatively recently, and is largely ignored in other areas(Zinn, 2008). Approaches that consider past emotional experiences are imperative in making reasonable and sound decisions (Baumeister, Dewall, \& Zhang, 2007, p. 16). Predominantly, this refers to taking actions based on anticipated emotion. A strong emotional connection to risk exists, which can cloud an individual's capacity to identify the probabilities of certain scenarios. The focus is instead on possible outcomes, and the dread factor associated with them. 'Dread' is addressed considerably in psychological risk perception theories, and a strong emotional feeling of dread is one of the largest factors in propelling an individual to act upon a risk (Baumeister et al., 2007, p. 20). The emotional feeling of 'dread' is typically associated with personal impacts, impacts on future generations and catastrophic widespread risks (Baumeister et al., 2007, p. 261). Emotions that indicate a positive or negative feeling to an individual are key factors in effective decision making in risk assessments (Baumeister et al., 2007).

\section{Central Theme of Risk Approaches}

There is significant literature in the field on the gap between expert risk perceptions and that of the public, creating a sense of scientific and social rationality that are constantly at odds (Beck, 1992, p. 30). The public consider elements of uncertainty, especially those associated with the feeling of dread and catastrophic events, whereas experts are far more likely to consider factors like likelihood of harm or expected mortality (Slovic, 1993, p. 691).

... [E]xpert knowledge and public knowledge are conditional- each reflects the underlying social relations and implicit assumptions of the various actors (Golding, 1992, p. 24).

A risk assessor who argues that risk is always strictly objective will struggle to understand a layperson's assessment of risk, and thus give it less weight or credibility than that of an expert. Self describes this as a key disadvantage of a technical and econocratic approach (Self, 1975). There is ample evidence to suggest that risk cannot be seen as purely objective, or positivist, and that people do not behave as purely rational actors (Douglas, 1997). Proponents of the psychological theory contend that human judgement plays a large role in interpreting scientific data, so when data is analysed and presented, it is by its very nature biased, making an 'objective' risk analysis even less 'objective' (Fischhoff et al., 1978).

Risk does not exist 'out there', independent of our minds and cultures, waiting to be measured. Human beings have invented the concept of 'risk' to help them understand and cope with the dangers and uncertainties of life (Slovic, 1992, p. 119).

Despite contending that there is bias at work in the development of risk perceptions, one should not presuppose that these risk perceptions are illegitimate value judgements about what is deemed acceptable (Shrader-Frechette, 1991, p. 54). Expert risk perceptions are not necessarily more valuable than a layperson's risk perception. The layperson's analysis of risk can reveal a significant amount of value in understanding public values and concerns, and in answering Beck's question of 'how do we want to live?' As such, public risk perceptions are not necessarily wrong (Golding, 1992). To the contrary, they show the social and cultural contexts of the day, which can be useful in devising appropriate risk management strategies. These risk perceptions can be used in synergy with experts' own risk perceptions to find commonalities and strategies for striking a balance between the two.

These psychological factors present a convincing theory on how risk is assessed by individuals, particularly in considering emotive drivers, a key element that is largely ignored by the other perspectives on risk. There is significant research in the field suggesting that these psychological elements have a large impact upon risk and its social construction (Slovic, 1992, p. 120); (Kahneman \& Tversky, 1979); (Gregory \& Mendelsohn, 1993); (Loewenstein, Weber, Hsee, \& Welch, 2001); (Slovic, 1999).

The psychological reading of risk is however not without criticism. It has been argued that the approach is difficult to implement directly in risk practices and policies (Renn, 1992, p. 190). Furthermore, Plough and Krimsky argue that it is challenging to find a common denominator in risk perception when the focus is on the individual's views, which can be extremely varied in nature (Plough \& Krimsky, 1987). Despite these criticisms, 
efforts to understand risk perception and its influencing factors offer key insights into the real concerns and values of individuals and population groups. These approaches are often completely overlooked by technical approaches to risk (which are used in many organisations), limiting the value of these assessments. Understanding and incorporating other forms of approaching risk outside the traditional technical risk approach could provide further valuable insight in how risk varies between risk assessors. The water industry predominantly utilises the technical risk approach and therefore, could be disregarding other influences in developing risk attitudes. This provides a limited approach to risk, that may result in widely varied risk assessments in the water industry.

\section{Conclusion}

The discussion on the sociological, cultural and psychological perspectives on risk provides a glimpse into a field that has not been utilised in practice. Despite many decades of research into risk, these risk approaches have been ignored in the water industry, and not utilised by government authorities. The overwhelming predominance of the use of the technical perspective in assessing risks throughout industry and business, could ignore the very nature of human-based decision making, in that it is not devoid of social and psychological influence. Through understanding the other risk approaches, government and industry could benefit through employing a holistic, interdisciplinary approach to risk. The approaches provide insight into differing perspectives that may be adopted for use, especially as there is increasing criticism of technical risk assessments, and in so doing, address key aspects of human resolutions such as the influence of social, cultural and psychological that could serve to render a differing risk outcome.

\section{References}

Alhakami, A. S., \& Slovic, P. (1994). A psychological study of the inverse relationship between perceived risk and perceived benefit. Risk Analysis: An Official Publication Of The Society For Risk Analysis, 14(6), 1085-1096.

Anderson, A. (1997). Media, Culture and the Environment. London: UCL Press.

Baumeister, R. F., Dewall, N., \& Zhang, L. (2007). Do Emotions Improve or Hinder the Decision Making Process? In K. D. Vohs, R. F. Baumeister, \& G. F. Loewenstein (Eds.), Do Emotions Help or Hurt Decision making: a hedgefoxian perspective (pp. 11-32). New York: Russell Sage Foundation.

Beck, U. (1992). Risk society: towards a new modernity. London: Sage Publications.

Beck, U. (1999). World risk society. Malden, Mass: Polity Press.

Beck, U. (2006). Living in the world risk society: a hobhouse memorial public lecture given on Wednesday 15 February 2006 at the London School of Economics. Economy and Society, (3), 329.

Douglas, M. (1992). Risk and Blame: Essays in Cultural Theory. London: Routledge.

Douglas, M. (1997). The Depoliticization of Risk. In R. Ellis \& M. Thompson (Eds.), Culture Matters: essays in honor of Aaron Wildavsky. Boulder, Colo.: Westview Press.

Environmental Protection Agency Victoria. (2004). Risk-Based Assessment of Ecosystem Protection in Ambient Waters. (Publication 961). Melbourne Retrieved from http://www.epa.vic.gov.au/ /media/Publications/ 961.pdf.

Fischhoff, B. (1975). Hindsight Not Equal To Foresight - Effect Of Outcome Knowledge On Judgment Under Uncertainty. Journal of Experimental Psychology-Human Perception and Performance, 1(3), 288-299. https://doi.org/10.1037//0096-1523.1.3.288

Fischhoff, B., Slovic, P., Lichtenstein, S., Read, S., \& Combs, B. (1978). How Safe Is Safe Enough Psychometric Study Of Attitudes Towards Technological Risks And Benefits. Policy Sciences, 9(2), 127-152. https://doi.org/10.1007/bf00143739

Golding, D. (1992). A Social and Programmatic History of Risk Research. In S. Krimsky \& D. Golding (Eds.), Social Theores of Risk (pp. 23-52). Westport, CT: Praeger Publishers.

Gregory, R., \& Mendelsohn, R. (1993). Perceived risk, dread, and benefits. Risk Analysis; (United States), 259.

Kahneman, D., \& Tversky, A. (1979). Prospect Theory: An Analysis of Decision under Risk. The Econometric Society, (2), 263.

Loewenstein, G. F., Weber, E. U., Hsee, C. K., \& Welch, N. (2001). Risk as feelings. Psychological Bulletin, 127(2), 267-286. https://doi.org/10.1037//0033-2909.127.2.267 
Lupton, D. (2013). Risk (2nd ed.). New York and Oxon: Routledge.

Pielke, R. A. (2007). The honest broker : making sense of science in policy and politics / Roger A. Pielke, Jr: Cambridge ; New York : Cambridge University Press.

Plough, A., \& Krimsky, S. (1987). The Emergence of Risk Communication Studies: Social and Political Context. Science, Technology and Human Values, 12, 4-10.

Rayner, S. (1992). Cultural Theory and Risk Analysis. In S. Krimsky \& D. Golding (Eds.), Social Theories of Risk (pp. 83-116). Westport, CT: Praeger Publishers.

Renn, O. (1992). Concepts of Risk: A Classification. In S. Krimsky \& D. Golding (Eds.), Social Theories of Risk (pp. 53-82). Westport, CT: Praeger Publishers.

Self, P. (1975). Econocrats and the policy process : the politics and philosophy of cost-benefit analysis. London: Macmillan.

Shrader-Frechette, K. S. (1991). Risk and rationality : philosophical foundations for populist reforms. Berkeley University of California Press.

Siegrist, M., Cvetkovich, G., \& Roth, C. (2000). Salient value similarity, social trust, and risk/benefit perception. Risk Analysis, 20(3), 353-362. https://doi.org/10.1111/0272-4332.203034

Slovic, P. (1992). Perception of Risk. In S. Krimsky \& D. Golding (Eds.), Social Theories of Risk (pp. 117-153). Westport, CT: Praeger Publisher.

Slovic, P. (1993). Perceived risk, trust, and democracy. Risk Analysis: An International Journal, 675.

Slovic, P. (1999). Trust, emotion, sex, politics, and science: Surveying the risk-assessment battlefield (Reprinted from Environment, ethics, and behavior, pg 277-313, 1997). Risk Analysis, 19(4), 689-701. https://doi.org/ 10.1111/j.1539-6924.1999.tb00439.x

Starr, C. (1969). Social Benefit versus Technological Risk. Science, 165, 1232-1238.

Taylor-Gooby, P., \& Zinn, J. (2006). Risk in social science. Oxford ; New York: Oxford University Press.

Victorian Managed Insurance Authority. (2016). The Victorian Government Risk Management Framework Practice Guide. Retrieved from https://www.vmia.vic.gov.au/risk/risk-tools/risk-management-guide

Von Neumann, J., \& Morgenstern, O. (2007). Theory of games and economic behavior. [electronic resource]. Princeton: Princeton University Press.

Zinn, J. (2008). Heading into the Unknown: Everyday Strategies for Managing Risk and Uncertainty. Health, Risk and Society, 10(5). https://doi.org/10.1080/13698570802380891

Zinn, J. (2009). Social Theories of Risk and Uncertainty. [electronic resource] : An Introduction. Hoboken: Wiley.

\section{Notes}

Note 1. Risk is taken as 'the possibility that something unpleasant and unwelcome will happen' ("Oxford Dictionaries." Oxford University Press, Accessed via http://www.oxforddictionaries.com/definition/english/risk. [16 August 2016]). Risk is always in the future, and should be noted that it differs from 'uncertainty' which doesn't imply an unpleasant consequence. There will be some level of uncertainty within a risk, however the term cannot be used interchangeably with uncertainty.

Note 2. Lupton discusses the historical aspect of the term, highlighting that its earliest use dates back to only the $18^{\text {th }}$ Century. In addition to this, the very meaning of the term has changed significantly from an occurrence that is fated to happen due to gods or a higher power to the idea that it is a human-caused issue that can controlled by humans as well (Lupton, 2013, p. 11).

\section{Copyrights}

Copyright for this article is retained by the author(s), with first publication rights granted to the journal.

This is an open-access article distributed under the terms and conditions of the Creative Commons Attribution license (http://creativecommons.org/licenses/by/4.0/). 